\title{
Infección respiratoria aguda en primera infancia, estudio de casos y controles, Suaza 2011
}

\author{
Acute respiratory infection in early childhoo, case \\ study and controls, Suaza 2011
}

Cheylans Duran ${ }^{1}$, Yuliette Tribiño ${ }^{2}$

Palabras clave: infección del tracto respiratorio, infección, enfermedades del tracto respiratorio, infecciones bacterianas y micosis.

Key words: respiratory tract infection, infection, respiratory tract diseases, bacterial infections and mycosis.

\section{Resumen}

La Infección Respiratoria Aguda (IRA) se encuentra dentro de las cinco primeras causas de morbilidad en primera infancia en el municipio de Suaza, Huila. Aunque se realizan acciones en salud pública encaminadas a la protección de la madre y el menor, no se presenta una disminución estadísticamente significativa en la incidencia de la patología. Con el fin de establecer los factores asociados a la presentación de la enfermedad, se realizó un estudio de casos y controles prospectivo entre marzo y abril de 2011.

Los casos fueron niños menores de cinco años con diagnóstico de IRA según lo estipulado en el protocolo de vigilancia en salud pública para IRA y los criterios del CIE 10; los controles eran niños sin diagnóstico de IRA en el mismo grupo de edad, vecinos de los casos. Se estudiaron 176 menores de cinco años (88 casos y 88 controles), encontrándose mayor incidencia de la patología en la zona rural.

No se hallaron factores de riesgo asociados a IRA y contrario a lo esperado, se presentaron como factores protectores: ingreso menor a un salario mínimo legal mensual (OR= 0,49; IC95 \%: 0,24-0,97; ji = 4,24; $\mathrm{p}=0,039)$, y bajo peso del niño al nacer (OR= 0,48; IC95 \%:0,26- 0,87; ji = 5,87; $\mathrm{p}=$ $0,01)$. Aunque no se encontraron factores de riesgo para la presentación de la patología, no se puede asegurar que no existan, por esto se requieren nuevos estudios, donde se prolongue el tiempo de investigación y aumente el tamaño de muestra.

\footnotetext{
Abstract

Acute respiratory infection (ARI) is within the five leading causes of morbidity in early childhood in the town of Suaza, Huila. Despite efforts in public health aim at protecting the mother and the child have been made, there is not a statistically significant decrease in the incidence of the disease. In order to establish the factors associated with the disease outbreak, it was conducted a case study and prospective controls between March and April, 2011.

The cases were children under five years old with ARI diagnosis as stipulated in the protocol for public health surveillance for ARI and ICD10 criteria; controls were children without ARI diagnosis, in the same age group, neighbors of the cases. 176 children under five years old were studied, (88 cases and 88 controls). It was found higher incidence of the disease in rural areas.
}

1 Especialista en Epidemiologia, Facultad de Salud, Universidad Surcolombiana, Hospital Universitario de la Samaritana, Bogotá, Colombia.

2 Especialista en Epidemiologia, Facultad de Salud, Universidad Surcolombiana, Hospital Universitario de Neiva, Colombia.

Correspondencia: Correo electrónico: lila_522@hotmail.com

Recibido: 1 de agosto de 2011

Aceptado: 28 de octubre de 2011 
There were no risk factors associated with ARI, and contrary to expectations, the following were found as protective factors: income below the legal minimum wage (OR $=0.49,95 \%$ CI 0.24 to 0.97 , chi $=4.24$, $\mathrm{P}=0.039)$, and low infant weight at birth $(\mathrm{OR}=0.48,95 \% \mathrm{CI} 0.26$ to 0.87 , chi $=5.87, P=0.01$ ). Although there were no found risk factors for the disease outbreak, there is no assurance of their absence, so further longer studies with a bigger sample size are required.

\section{Introducción}

La infección respiratoria es una patología de presentación aguda, y el manejo inadecuado genera el inicio enfermedades como otitis media y neumonía, siendo la neumonía la principal causa de impacto en la morbi-mortalidad de la primera infancia en países en vía de desarrollo(1).

Existen estudios que evidencian los múltiples factores que influyen en el desarrollo de la Infección Respiratoria Aguda (IRA), tales como: factores socioeconómicos (edad, género, estrato, condiciones de vivienda)(3), biológicos (peso al nacer, estado nutricional, lactancia materna, PAI completo), ambientales (clima, polución atmosférica) y de conocimiento de la madre ${ }^{4}$.

Por ser considerada en Colombia como un problema de salud pública, debido a la alta incidencia en menores de 5 años ${ }^{(2)}$, se han realizado investigaciones que han ayudado a la identificación de los factores de riesgo que influyen en la presentación de la enfermedad y sus complicaciones, de esta manera se han implementado acciones para la intervención oportuna, con el fin de generar protección al menor ${ }^{(4)}$.

En el Huila, es una patología de notificación obligatoria al SIVIGILA por encontrarse en el primer renglón de morbilidad ${ }^{(5)}$; por otro lado en el municipio de Suaza está dentro de las cinco primeras causas de morbilidad por encima de la $\operatorname{EDA}^{(6)}$.

El estudio tenía como finalidad la identificación de los factores de riesgo que inciden en la presentación de la infección respiratoria aguda en menores de cinco años en el municipio de Suaza, pero al realizar el análisis de los datos se encontraron factores protectores epidemiológicamente significativos que no ayudaron a solucionar el problema planteado inicialmente.

\section{Materiales y métodos}

Se realizó un estudio analítico de casos y controles prospectivo en un bimestre, donde se comparan las posibles exposiciones para la presentación de la enfermedad de los individuos enfermos (casos) y no enfermos (controles), se calcula la proporción de los casos y los controles (odds ratio).

\section{Definición del caso}

Como caso de IRA fue tomado el niño menor de cinco años, residente en el municipio de Suaza, que ingrese a la ESE Hospital Nuestra Señora de Fátima por Consulta externa y urgencias, presente diagnóstico clínico presuntivo de IRA según lo estipulado en protocolo Vigilancia de Salud Pública para Infección Respiratoria Aguda ${ }^{(7)}$ y los criterios del CIE 10.

\section{Definición del control}

Como caso control se aceptó a niño menor de cinco años, sin diagnóstico de IRA, vecino del caso de IRA en cinco casas a la redonda. En caso de ausencia de un niño menor de cinco años en la delimitación estipulada, se tomó la casa consecutiva hasta que un niño con los criterios mencionados fue hallado.

\section{Criterios de exclusión}

Se excluyeron a los niños menores de cinco años, que presenten síntomas de IRA sin diagnóstico médico de la ESE, Hospital Nuestra Señora de Fátima. 


\section{Fuentes de información}

La fuente de información utilizada fue una encuesta aplicada a las madres o acudientes del menor que fueron seleccionados como caso o control. El instrumento fue revisado por un médico pediatra quien confirmó la existencia de todos los factores referenciados en la presentación de IRA a los menores de cinco años. Para la aplicación de la encuesta se entrenaron dos auxiliares de enfermería, que realizaron el diligenciamiento de la encuesta y escogencia del control, además se encargaron de evitar los diferentes sesgos en el estudio, enfatizando en realizar de igual manera las preguntas para casos y controles, sin dirigir las respuestas de los entrevistados.

\section{Recolección de la información}

A los menores que participaron en el estudio se les aplicó una encuesta previamente validada por un pediatra, escogiéndose las siguientes variables:

1. Condiciones sociodemográficas: edad, género, seguridad social, procedencia, edad de la madre, escolaridad de la madre e ingreso mensual.

2. Condiciones de vivienda: cuántas personas viven, cuartos, tipo de materiales de pisos y paredes, servicios públicos, materiales utilizados para cocinar.

3. Condiciones medioambientales: consumo de tabaco o cigarrillo, forma de eliminación de las basuras, número de niños residentes en la vivienda, asistencia a la guardería.

4. Condiciones familiares: actividades de la madre antes y durante el embarazo; fumar durante el embarazo, presencia de asma, número de embarazos previos, meses de nacimiento y tipo de parto del menor.

5. Estado nutricional y programa ampliado de inmunizaciones: peso del niño; si hubo lactancia materna y su duración; vacunas adecuadas para la edad.

6. Comorbilidades crónicas y comportamentales: el niño presenta alguna enfermedad crónica, lavado de manos en presencia de gripa, utilización de medios de protección contra el frío.

\section{Creación de base de datos}

Se creó una base de datos en Excel 2003, donde se almacenó la información recogida en la encuesta, según las variables anteriormente descritas, para su posterior análisis estadístico.

\section{Comité de Ética Institucional}

La realización de la investigación se basó en la aprobación y firma de un consentimiento informado a los padres o acudientes del menor, sin que el hecho de no participar les signifique algún deterioro en la atención médica, donde se garantiza la absoluta confidencialidad en el manejo de los cuestionarios, los cuales después de grabarse en base de datos, serán destruidos.

La propuesta de investigación se presentó al comité de Ética de la Facultad de Salud de la Universidad Surcolombiana para su aprobación y evaluación de la encuesta, buscando posibles afectaciones de tipo ético; por ser un cuestionario que se basa en los factores de riesgo encontrados en el marco conceptual, donde no invaden en ningún momento la privacidad de la madre o el niño(a), se clasifica esta investigación como estudio sin riesgo según lo estipulado en la Resolución 8430 de 1993, por el Ministerio de Salud.

\section{Análisis de los datos}

El programa estadístico utilizado fue el SPSS versión 18.01, se realizó análisis univariado, con el fin de caracterizar la población y determinar la incidencia de la patología en el casco urbano y zona rural, seguido de un análisis bivariado para establecer asociación entre las variables estudiadas y la presentación de IRA, por lo cual se calculó el odd ration (OR) y sus intervalos de confianza del 95\%. Para facilitar el análisis, las variables se agruparon en categorías descritas en la recolección de la información, identificando aquellas que tuvieron una asociación con un valor de $\mathrm{p}<0,05$.

Las variables dicotómicas se dejaron como tal y algunas se dicotomizaron buscando que 
su agrupamiento alcanzara el factor de riesgo para presentar infección respiratoria aguda. Por otro lado, se utilizó la prueba de $\mathrm{chi}^{2}$, para probar diferencias de proporciones en análisis y verificación de la hipótesis.

Como no se presentaron asociaciones de factores de riesgo para la presentación de la enfermedad, se omitió la realización del análisis multivariado de regresión logística.

\section{Resultados}

La recolección de los datos se realizó en los meses de marzo y abril de 2011. La población total para el análisis del estudio fue de 88 casos, cada uno con su respectivo control donde se cumplieron criterios de inclusión y exclusión.

Según lo encontrado en el grupo etáreo, el 40,9\% están en edades entre los siete y doce meses. Los menores de seis meses presentaron el menor porcentaje con un 9,1. El sexo masculino fue predominante en las encuestas con un $54 \%$; el $91,5 \%$ de la población está en el régimen subsidiado, y en menor proporción el $0,6 \%$ de la población encuestada aparece como desplazado. En cuanto a la procedencia, el $72,7 \%$ se encuentra en la zona rural (Tabla 1).

Tabla 1. Características sociodemográficas de los niños.

\begin{tabular}{|c|c|c|c|c|c|c|}
\hline & \multirow{2}{*}{ Frecuencia } & \multicolumn{2}{|c|}{ Caso } & \multicolumn{2}{|c|}{ Control } & \multirow{2}{*}{ Porcentaje } \\
\hline & & $\mathrm{N}^{\mathrm{o}}$ & $\%$ & $\mathrm{~N}^{\mathrm{o}}$ & $\%$ & \\
\hline \multicolumn{7}{|l|}{ Grupo etáreo } \\
\hline $1-6$ & 16 & 8 & 4,5 & 8 & 4,5 & 9,1 \\
\hline $7-12$ & 72 & 39 & 22,1 & 33 & 18,7 & 40,9 \\
\hline $13-24$ & 42 & 21 & 11,9 & 21 & 11,9 & 23,9 \\
\hline $25-36$ & 19 & 7 & 3,9 & 12 & 6,8 & 10,8 \\
\hline $37-50$ & 27 & 13 & 7,3 & 14 & 7,9 & 15,3 \\
\hline Total & 176 & 88 & 50 & 88 & 50 & 100,0 \\
\hline \multicolumn{7}{|l|}{ Género } \\
\hline Femenino & 81 & 39 & 22,1 & 42 & 23,8 & 46,0 \\
\hline Masculino & 95 & 49 & 27,8 & 46 & 26,1 & 54,0 \\
\hline Total & 176 & 88 & 50,0 & 88 & 50,0 & 100,0 \\
\hline \multicolumn{7}{|c|}{ Seguridad social } \\
\hline Contributivo & 8 & 4 & 2,2 & 4 & 2,2 & 4,5 \\
\hline Subsidiado & 161 & 83 & 47,1 & 78 & 44,3 & 91,5 \\
\hline Otro & 6 & 1 & 0,5 & 5 & 2,8 & 3,4 \\
\hline Desplazado & 1 & 0 & 0 & 1 & 0,5 & 0,6 \\
\hline Total & 176 & 88 & 50,0 & 88 & 50,0 & 100,0 \\
\hline \multicolumn{7}{|l|}{ Procedencia } \\
\hline Urbano & 48 & 24 & 13,6 & 24 & 13,6 & 27,3 \\
\hline Rural & 128 & 64 & 36,3 & 64 & 36,3 & 72,7 \\
\hline Total & 176 & 88 & 50 & 88 & 50 & 100,0 \\
\hline
\end{tabular}


Tabla 2. Características sociodemográficas de la madre.

\begin{tabular}{|c|c|c|c|c|c|c|}
\hline & \multirow{2}{*}{ Frecuencia } & \multicolumn{2}{|c|}{ Caso } & \multicolumn{2}{|c|}{ Control } & \multirow{2}{*}{ Porcentaje } \\
\hline & & $\mathrm{N}^{\mathrm{o}}$ & $\%$ & $\mathrm{~N}^{\mathrm{o}}$ & $\%$ & \\
\hline \multicolumn{7}{|l|}{ Grupo etáreo } \\
\hline$<20$ & 36 & 17 & 9,6 & 19 & 10,7 & 20,5 \\
\hline $20-30$ & 92 & 49 & 27,8 & 43 & 24,4 & 52,3 \\
\hline $30-40$ & 39 & 17 & 9,6 & 22 & 12,5 & 22,2 \\
\hline$>40$ & 9 & 5 & 2,8 & 4 & 2,2 & 5,1 \\
\hline Total & 176 & 88 & 50,0 & 88 & 50,0 & 100,0 \\
\hline \multicolumn{7}{|l|}{ Estudios aprobados } \\
\hline Analfabeta & 1 & 0 & 0 & 1 & 0,5 & 0,6 \\
\hline Primaria & 61 & 30 & 17,0 & 31 & 17,6 & 34,7 \\
\hline Secundaria & 67 & 32 & 18,1 & 35 & 19,8 & 38,1 \\
\hline Media académica & 43 & 24 & 13,6 & 19 & 10,7 & 24,4 \\
\hline Pregrado y postgrado & 4 & 2 & 1,1 & 2 & 1,1 & 2,3 \\
\hline Total & 176 & 88 & 50 & 88 & 50 & 100,0 \\
\hline
\end{tabular}

El 52,3\% de las madres están en edad promedio de 21-30 años; los estudios aprobados están en su mayoría en media académica con el $38,1 \%$ seguido del $34,7 \%$ con básica primaria (Tabla 2); el 73,9\% subsiste con un ingreso menor al salario mínimo legal (Gráfico 1).

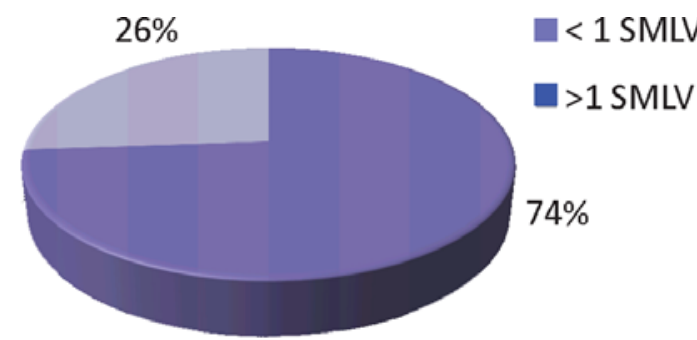

Figura 1. Descripción porcentual de ingreso mensual.

Para hacinamiento se toma como línea base el residir tres o más personas por habitación, teniendo en cuenta lo anteriormente descrito se encuentra que: el $45,4 \%$ residen de 1 a 4 personas, seguido del
$44,4 \%$ con 5 a 8 personas por casa, las viviendas en su mayoría con un $78,4 \%$ presentan de 1 a 3 cuartos. El grado de hacinamiento de las viviendas corresponde al $68,2 \%$ (Tabla 3).

Los datos hallados muestran que un $61.9 \%$ de las viviendas presentan pisos de cemento y el $65,3 \%$ de las casas presentan paredes de bahareque. Por otro lado, se observa que el $21,5 \%$ de los hogares no presentan alcantarillado, es de resaltar que el $6,8 \%$ no presenta ningún tipo de servicio público. En cuanto a la preparación de alimentos, el 39,2\% de los encuestados cocinan con leña, y el 23,8\% con leña y gas. Al analizar cuál es el método de eliminación de basuras, se encuentra que el 46,5\% la recogen los servicios de aseo, seguida de un $22,1 \%$ que la queman y arrojan al patio $\mathrm{u}$ otro terreno cercano (Tabla 4 ).

En su mayoría de 1 a 3 niños residen en una misma vivienda con el $80,7 \%$, en general, los niños no asisten a la guardería con un $78,9 \%$; de los encuestados solo el $18,8 \%$ 
Tabla 3. Viviendas en hacinamiento.

\begin{tabular}{|c|c|c|c|c|c|c|}
\hline & \multirow{2}{*}{ Frecuencia } & \multicolumn{2}{|c|}{ Caso } & \multicolumn{2}{|c|}{ Control } & \multirow{2}{*}{ Porcentaje } \\
\hline & & $\mathrm{N}^{\mathrm{o}}$ & $\%$ & $\mathrm{~N}^{\mathrm{o}}$ & $\%$ & \\
\hline \multicolumn{7}{|c|}{$\begin{array}{l}\text { Número de personas } \\
\text { que residen }\end{array}$} \\
\hline $1-4$ & 80 & 42 & 23,8 & 38 & 21,5 & 45,4 \\
\hline $5-8$ & 78 & 37 & 21,0 & 41 & 23,2 & 44,4 \\
\hline $9-12$ & 15 & 7 & 3,9 & 8 & 4,5 & 8,5 \\
\hline $13-16$ & 2 & 1 & 0,5 & 1 & 0,5 & 1,1 \\
\hline $17-20$ & 1 & 1 & 0,5 & 0 & 0 & 0,6 \\
\hline Total & 176 & 88 & 50,0 & 88 & 50,0 & 100,0 \\
\hline \multicolumn{7}{|c|}{ Número de cuartos } \\
\hline $1-3$ & 138 & 66 & 37,5 & 72 & 40,9 & 78,4 \\
\hline $4-6$ & 38 & 22 & 12,5 & 16 & 9,0 & 21,6 \\
\hline Total & 176 & 88 & 50 & 88 & 50 & 100,0 \\
\hline
\end{tabular}

Tabla 4. Características medioambientales de la vivienda.

\begin{tabular}{|c|c|c|c|c|c|c|}
\hline & \multirow{2}{*}{ Frecuencia } & \multicolumn{2}{|c|}{ Caso } & \multicolumn{2}{|c|}{ Control } & \multirow{2}{*}{ Porcentaje } \\
\hline & & $\mathrm{N}^{\circ}$ & $\%$ & $\mathrm{~N}^{\mathrm{o}}$ & $\%$ & \\
\hline \multicolumn{7}{|l|}{ Piso } \\
\hline Baldosa & 13 & 7 & 3,9 & 6 & 3,4 & 7,3 \\
\hline Cemento & 109 & 55 & 31,2 & 54 & 30,6 & 61,9 \\
\hline Madera & 5 & 2 & 1,1 & 3 & 1,7 & 2,8 \\
\hline Tierra - arena & 49 & 24 & 13,6 & 25 & 14,2 & 27,8 \\
\hline Total & 176 & 88 & 50,0 & 88 & 50,0 & 100,0 \\
\hline \multicolumn{7}{|l|}{ Paredes } \\
\hline Bloque & 58 & 26 & 14,7 & 32 & 18,1 & 33,0 \\
\hline Bareque & 115 & 61 & 34,6 & 54 & 30,6 & 65,3 \\
\hline Madera & 3 & 1 & 0,5 & 2 & 1,1 & 1,7 \\
\hline Total & 176 & 88 & 50,0 & 88 & 50,0 & 100,0 \\
\hline
\end{tabular}


Continuación tabla 4.

\begin{tabular}{|c|c|c|c|c|c|c|}
\hline & \multirow{2}{*}{ Frecuencia } & \multicolumn{2}{|c|}{ Caso } & \multicolumn{2}{|c|}{ Control } & \multirow{2}{*}{ Porcentaje } \\
\hline & & $\mathrm{N}^{\mathrm{o}}$ & $\%$ & $\mathrm{~N}^{\mathrm{o}}$ & $\%$ & \\
\hline \multicolumn{7}{|l|}{ Servicios públicos } \\
\hline Energía & 33 & 16 & 9,0 & 17 & 9,6 & 18,7 \\
\hline Alcantarillado & 38 & 20 & 11,3 & 18 & 10,2 & 21,5 \\
\hline Acueducto & 43 & 19 & 10,7 & 24 & 13,6 & 24,4 \\
\hline Gas natural & 5 & 2 & 1,1 & 3 & 1,7 & 2,8 \\
\hline Sin energía & 43 & 24 & 13,6 & 19 & 10,7 & 24,4 \\
\hline Sin alcantarillado & 1 & 1 & 0,5 & 0 & 0 & 0,5 \\
\hline Sin acueducto & 1 & 0 & 0 & 1 & 0,5 & 0,5 \\
\hline Sin servicios & 12 & 6 & 3,4 & 6 & 3,4 & 6,8 \\
\hline Total & 176 & 88 & 50,0 & 88 & 50,0 & 100,0 \\
\hline \multicolumn{7}{|l|}{ Cocinar } \\
\hline Leña & 69 & 33 & 18,7 & 36 & 20,4 & 39,2 \\
\hline Estufa a gas & 62 & 32 & 18,1 & 30 & 17,0 & 35,2 \\
\hline Estufa eléctrica & 3 & 2 & 1,1 & 1 & 0,5 & 1,7 \\
\hline Leña y gas & 42 & 21 & 11,9 & 21 & 11,9 & 23,8 \\
\hline Total & 176 & 88 & 50,0 & 88 & 50,0 & 100,0 \\
\hline \multicolumn{7}{|l|}{ Eliminación de basura } \\
\hline Servicio aseo & 82 & 41 & 23,2 & 41 & 23,2 & 46,5 \\
\hline Entierran & 4 & 2 & 1,1 & 2 & 1,1 & 2,2 \\
\hline Queman & 39 & 19 & 10,7 & 20 & 11,3 & 22,1 \\
\hline Tiran al patio & 9 & 4 & 2,2 & 5 & 2,8 & 5,1 \\
\hline Río & 29 & 14 & 7,9 & 15 & 8,5 & 16,4 \\
\hline Otra forma & 4 & 2 & 1,1 & 2 & 1,1 & 2,2 \\
\hline Patio y río & 1 & 1 & 0,5 & 0 & 0 & 0,5 \\
\hline Servicio aseo y queman & 7 & 4 & 2,2 & 3 & 1,7 & 3,9 \\
\hline Entierran y patio & 1 & 1 & 0,5 & 0 & 0 & 0,5 \\
\hline Total & 176 & 88 & 50 & 88 & 50 & 100,0 \\
\hline
\end{tabular}


están en contacto con personas que fuman tabaco (Tabla 5).

Con respecto al proceso de embarazo de las madres de los niños tomados para la investigación, solo 5,1\% fumaron durante este tiempo; no hubo presencia de asma en un $94,3 \%$ y más de 3 embarazos previos corresponden al 18,2 de las madres encuestadas (Tabla 6).

El 10,8\% de los niños nacieron pre término y en menor proporción con un $26,7 \%$ el nacimiento fue por cesárea (Tabla 7).

Por encima del 50\% los niños tuvieron un peso adecuado al nacer, el $47,7 \%$ se encontró según la tabla de crecimiento y desarrollo igual o por encima del percentil 50; el $50 \%$ tomaron leche materna de 0 a 6 meses y en los mismos meses el 93,8\% tomaron leche materna exclusiva (Tabla 8).

Al revisar el carné de vacunas se encuentra que el $7,4 \%$ de los niños no presentan programa ampliado de inmunizaciones completo para la edad (Figura 2).

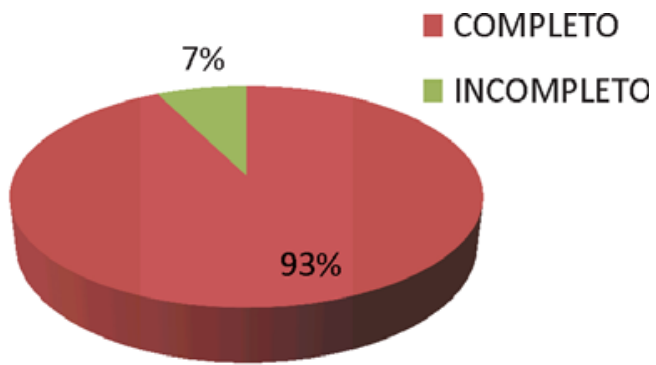

Figura 2. Porcentaje de vacunación en niños.

De los niños encuestados el 3,5 presenta algún tipo de comorbilidad, tales como: asma, problemas cardiacos y síndrome de Down (Tabla 9).

Se observa que el 26,1 de las personas encuestadas no se lavan las manos en caso de presentación de gripe, además que el $51,1 \%$ no protege al niño cuando está lloviendo o hace frío (Tabla 10).

El tener un ingreso menor a un salario mínimo legal mensual y que el niño presente un bajo peso al nacer, muestra valores de

Tabla 5. Características individuales del medio.

\begin{tabular}{|c|c|c|c|c|c|c|}
\hline & \multirow{2}{*}{ Frecuencia } & \multicolumn{2}{|c|}{ Caso } & \multicolumn{2}{|c|}{ Control } & \multirow{2}{*}{ Porcentaje } \\
\hline & & $\mathrm{N}^{\mathrm{o}}$ & $\%$ & $\mathrm{~N}^{\mathrm{o}}$ & $\%$ & \\
\hline \multicolumn{7}{|c|}{ Niños en la casa } \\
\hline $1-3$ & 142 & 72 & 40,9 & 70 & 39,7 & 80,7 \\
\hline $4-6$ & 33 & 15 & 8,5 & 18 & 10,2 & 18,8 \\
\hline$>6$ & 1 & 1 & 0,5 & 0 & 0 & 0,6 \\
\hline Total & 176 & 88 & 50,0 & 88 & 50,0 & 100,0 \\
\hline \multicolumn{7}{|c|}{ Guardería } \\
\hline $\mathrm{Si}$ & 37 & 18 & 10,2 & 19 & 10,7 & 21,0 \\
\hline No & 139 & 70 & 39,7 & 69 & 39,2 & 78,9 \\
\hline Total & 176 & 88 & 50,0 & 88 & 50,0 & 100,0 \\
\hline \multicolumn{7}{|c|}{ Tabaco } \\
\hline $\mathrm{Si}$ & 33 & 17 & 9,6 & 16 & 9,0 & 18,8 \\
\hline No & 143 & 71 & 40,3 & 72 & 40,9 & 81,3 \\
\hline Total & 176 & 88 & 50 & 88 & 50 & 100,0 \\
\hline
\end{tabular}


Tabla 6. Características comportamentales y antecedentes médicos de la madre.

\begin{tabular}{|c|c|c|c|c|c|c|}
\hline & \multirow{2}{*}{ Frecuencia } & \multicolumn{2}{|c|}{ Caso } & \multicolumn{2}{|c|}{ Control } & \multirow{2}{*}{ Porcentaje } \\
\hline & & $\mathrm{N}^{\mathrm{o}}$ & $\%$ & $\mathrm{~N}^{\mathrm{o}}$ & $\%$ & \\
\hline \multicolumn{7}{|c|}{ Fumar } \\
\hline $\mathrm{Si}$ & 9 & 5 & 2,8 & 4 & 2,2 & 5,1 \\
\hline No & 167 & 83 & 47,1 & 84 & 47,7 & 84,9 \\
\hline Total & 176 & 88 & 50,0 & 88 & 50,0 & 100,0 \\
\hline \multicolumn{7}{|c|}{ Asma } \\
\hline $\mathrm{Si}$ & 10 & 6 & 3,4 & 4 & 2,2 & 5,7 \\
\hline No & 166 & 82 & 46,5 & 84 & 47,7 & 94,3 \\
\hline Total & 176 & 88 & 50,0 & 88 & 50,0 & 100,0 \\
\hline \multicolumn{7}{|c|}{ Embarazos previos } \\
\hline $0-2$ & 144 & 77 & 43,7 & 67 & 38,0 & 81,8 \\
\hline $3-5$ & 26 & 8 & 4,5 & 18 & 10,2 & 14,8 \\
\hline $6-8$ & 6 & 3 & 1,7 & 3 & 1,7 & 3,4 \\
\hline Total & 176 & 88 & 50 & 88 & 50 & 100,0 \\
\hline
\end{tabular}

Tabla 7. Características de nacimiento del niño.

\begin{tabular}{|c|c|c|c|c|c|c|}
\hline & \multirow{2}{*}{ Frecuencia } & \multicolumn{2}{|c|}{ Caso } & \multicolumn{2}{|c|}{ Control } & \multirow{2}{*}{ Porcentaje } \\
\hline & & $\mathrm{N}^{\mathrm{o}}$ & $\%$ & $\mathrm{~N}^{\mathrm{o}}$ & $\%$ & \\
\hline \multicolumn{7}{|c|}{ Tipo de parto } \\
\hline Vaginal & 129 & 68 & 38,6 & 61 & 34,6 & 73,3 \\
\hline Cesárea & 47 & 20 & 11,3 & 27 & 15,3 & 26,7 \\
\hline Total & 176 & 88 & 50,0 & 88 & 50,0 & 100,0 \\
\hline \multicolumn{7}{|c|}{ Meses de gestación } \\
\hline 7 & 5 & 2 & 1,1 & 3 & 1,7 & 2,8 \\
\hline 8 & 14 & 4 & 2,2 & 10 & 5,6 & 8,0 \\
\hline 9 & 157 & 82 & 46,5 & 75 & 42,6 & 89,2 \\
\hline Total & 176 & 88 & 50 & 88 & 50 & 100,0 \\
\hline
\end{tabular}


Tabla 8. Características nutricionales.

\begin{tabular}{|c|c|c|c|c|c|c|}
\hline & \multirow{2}{*}{ Frecuencia } & \multicolumn{2}{|c|}{ Caso } & \multicolumn{2}{|c|}{ Control } & \multirow{2}{*}{ Porcentaje } \\
\hline & & $\mathrm{N}^{\mathrm{o}}$ & $\%$ & $\mathrm{~N}^{\mathrm{o}}$ & $\%$ & \\
\hline \multicolumn{7}{|l|}{ Peso del niño } \\
\hline Sin dato & 2 & 1 & 0,5 & 1 & 0,5 & 1,1 \\
\hline Bajo & 94 & 39 & 22,1 & 55 & 31,2 & 53,4 \\
\hline Adecuado & 80 & 48 & 27,2 & 32 & 18,1 & 45,4 \\
\hline Total & 176 & 88 & 50,0 & 88 & 50,0 & 100,0 \\
\hline \multicolumn{7}{|c|}{ Crecimiento y desarrollo } \\
\hline No sabe & 2 & 1 & 0,5 & 1 & 0,5 & 1,1 \\
\hline Adecuado & 84 & 47 & 26,7 & 37 & 21,0 & 47,7 \\
\hline Mal nutrición & 47 & 21 & 11,9 & 26 & 14,7 & 26,7 \\
\hline Desnutrición & 43 & 19 & 10,7 & 24 & 13,6 & 24,4 \\
\hline Total & 176 & 88 & 50,0 & 88 & 50,0 & 100,0 \\
\hline \multicolumn{7}{|c|}{ Leche materna } \\
\hline $0-6$ & 36 & 16 & 9,0 & 20 & 11,3 & 20,5 \\
\hline $6-12$ & 88 & 46 & 26,1 & 42 & 23,8 & 50,0 \\
\hline $13-24$ & 51 & 26 & 14,7 & 25 & 14,2 & 29,0 \\
\hline $25-36$ & 1 & 0 & 0 & 1 & 0,5 & 0,5 \\
\hline Total & 176 & 88 & 50,0 & 88 & 50,0 & 100,0 \\
\hline \multicolumn{7}{|c|}{ Leche materna exclusiva } \\
\hline $0-6$ & 165 & 84 & 47,7 & 81 & 46,0 & 93,8 \\
\hline $6-12$ & 10 & 3 & 1,7 & 7 & 3,9 & 5,7 \\
\hline $13-24$ & 1 & 1 & 0,5 & 0 & 0 & 0,5 \\
\hline Total & 176 & 88 & 50 & 88 & 50 & 100,0 \\
\hline
\end{tabular}

Tabla 9. Co-morbilidades del menor.

\begin{tabular}{|c|c|c|c|c|c|c|}
\hline & \multirow{2}{*}{ Frecuencia } & \multicolumn{2}{|c|}{ Caso } & \multicolumn{2}{|c|}{ Control } & \multirow{2}{*}{ Porcentaje } \\
\hline & & $\mathrm{N}^{\circ}$ & $\%$ & $\mathrm{~N}^{\mathrm{o}}$ & $\%$ & \\
\hline \multicolumn{7}{|c|}{ Enfermedades } \\
\hline Ninguna & 170 & 86 & 48,8 & 84 & 47,7 & 96,6 \\
\hline Asma & 4 & 2 & 1,1 & 2 & 1,1 & 2,3 \\
\hline Sind. Down & 1 & 0 & 0 & 1 & 0,5 & 0,6 \\
\hline Soplo & 1 & 0 & 0 & 1 & 0,5 & 0,6 \\
\hline Total & 176 & 88 & 50 & 88 & 50 & 100,0 \\
\hline
\end{tabular}


Tabla 10. Aspectos comportamentales de la familia.

\begin{tabular}{|c|c|c|c|c|c|c|}
\hline & \multirow{2}{*}{ Frecuencia } & \multicolumn{2}{|c|}{ Caso } & \multicolumn{2}{|c|}{ Control } & \multirow{2}{*}{ Porcentaje } \\
\hline & & $\mathrm{N}^{\mathrm{o}}$ & $\%$ & $\mathrm{~N}^{\mathrm{o}}$ & $\%$ & \\
\hline \multicolumn{7}{|l|}{ Lavado de manos } \\
\hline $\mathrm{Si}$ & 130 & 66 & 37,5 & 64 & 36,3 & 73,9 \\
\hline No & 46 & 22 & 12,5 & 24 & 13,6 & 26,1 \\
\hline Total & 176 & 88 & 50,0 & 88 & 50,0 & 100,0 \\
\hline \multicolumn{7}{|c|}{ Protección del niño al frío } \\
\hline Saco & 6 & 2 & 1,1 & 4 & 2,2 & 3,4 \\
\hline Saco y cobija & 90 & 43 & 24,4 & 47 & 26,7 & 51,1 \\
\hline Saco, cobija y ruana & 79 & 42 & 23,8 & 37 & 21,0 & 44,9 \\
\hline No lo protege & 1 & 1 & 0,5 & 0 & 0 & 0,6 \\
\hline Total & 176 & 88 & 50 & 88 & 55 & 100,0 \\
\hline
\end{tabular}

Tabla 11. Diferencias estadísticamente significativas para IRA.

\begin{tabular}{lccccccc}
\hline Factores identificados & Variable & Intervalo & Total & OR & IC 95\% & Chi2 & P \\
\hline \multirow{2}{*}{ Sociodemográficas } & Ingreso mensual & $<$ de 1 smlv & 130 & 0,49 & $0,24-0,97$ & 4,24 & 0,039 \\
& & $>$ de 1 smlv & 46 & & & & \\
\hline \multirow{2}{*}{ Nutricionales } & Peso del niño al nacer & Bajo & 96 & 0,48 & $0,26-0,87$ & 5,87 & 0,01 \\
& & Adecuado & 80 & & & & \\
\hline
\end{tabular}

OR $<1$ con un intervalo de confianza que no pasan por la unidad $y$ una $P$ menor a $\mathbf{0 , 0 5}$; de esta manera las variables se presentan como un probable factor protector disminuyendo la probabilidad de desarrollar enfermedad respiratoria aguda. Por otro lado, las otras características estudiadas no muestran un resultado epidemiológicamente significativo para factores de riesgo, por lo tanto no se puede validar ni rechazar la hipótesis presentada en la investigación (Tabla 11).

\section{Discusión}

En los meses de marzo y abril del 2011, en el hospital Nuestra Señora de Fátima, registró 106 casos en las áreas de consulta externa y urgencias por diagnóstico de IRA en menores de cinco años. Con el fin de disminuir el sesgo de información, cada semana, se realizaba desplazamiento a nivel domiciliario del caso, seleccionando el control y ejecutando aplicación del instrumento.

Recordando que la IRA es una patología de presentación aguda, por lo cual el hecho que al momento de la encuesta el niño no presente la enfermedad (control), no me está diciendo que los factores determinantes a los que está expuesto el menor no lo pongan en riesgo para un episodio futuro.

Aunque se buscó minimizar las debilidades en el estudio referente a la recolección de la información, se presentaron dificultades debido a las condiciones climáticas, dificultad de acceso vial y traslado del menor hacia otra vereda, de esta 
manera se obstaculiza a nivel operativo la investigación, presentando una pérdida de población de un $16,9 \%$, lo cual no es representativo para presentar un sesgo de selección.

Al realizar un análisis de la variables de estudio, se encuentra que la población menor de un año, esta por debajo del $50 \%$ atendido por patologías respiratorias, algo que se aleja un poco de lo encontrado por Jaimes ${ }^{(4)}$, donde más del $70 \%$ de la población estudiada, presentaba menos de 1 año, es de anotar que en el resultado de ambas investigaciones la característica similar es el pertenecer al género masculino, siendo un factor de riesgo para presentar patologías respiratorias, aumentando la probabilidad de asistencia médica.

Con respecto a los factores relacionados con la edad y escolaridad de la madre, no se encontró significancia estadística con la presencia de analfabetismo o el haber cursado algún tipo de escolaridad, Según Gómez $^{(8)}$ el $72,1 \%$ de las madres en edades entre 20 y 30 años presentan un mejor manejo de las enfermedades respiratorias debido a experiencia y buen manejo de la patología.

De igual manera tampoco se encuentra ningún tipo de relación en el tener más de 2 embarazos previos, en un estudio publicado en la revista BMC Public Health ${ }^{(9)}$, se encuentra que haber nacido de una madre con tres o más embarazos anteriores representaron en un $15,4 \%$ para factor de riesgo $(14,1$ a 17,0 en madres aborígenes y no aborígenes).

Además se encontró que la mayor asistencia médica fue de la zona rural. Por otro lado, es de anotar que según la base de datos del Hospital, se registraron 347 atenciones en consulta externa y urgencias a menores de cinco años en los meses de marzo y abril. Estas se dividen de la siguiente forma:

Casco urbano: de 116 atenciones, 32 fueron diagnosticadas con infección respiratoria aguda, presentando una incidencia de la enfermedad de un $27 \%$ en la población asistente.

Área rural: de 228 atenciones, 74 fueron diagnosticadas con infección respiratoria aguda, presentando una incidencia en la patología del 32\%.

Con estos resultados se concluye que la incidencia de la patología es mayor en la zona rural que en la cabecera municipal, presentándose relación de alguna manera con la menor población en la zona urbana según lo estipulado por las cifras DANE(10).

El vivir más de tres personas en una misma habitación se considera hacinamiento. Se encuentra que no tienen cuartos suficientes para el total de personas que residen en las viviendas. Es de resaltar que aunque se encuentra que la mayoría de la población reside de 1 a 8 personas por casa, y casi en igual proporción las viviendas constan de 1 a 3 habitaciones, la distribución de las habitaciones no es identificada en el instrumento, por lo tanto se hace difícil la conclusión de la variable.

Jaimes ${ }^{(4)}$, encontró que "cuando el menor comparte la cama con varias personas, el riesgo es 1,88 mayor (IC95\% 1,0-3,7) y el habitar más de 9 personas en una misma vivienda presenta un riesgo 1,82 mayor (IC $95 \% 1,0-3,51$. p. 0,022)».

Durante la etapa de gestación no se demostraron inconvenientes que pudieran afectar la salud del menor; a nivel de nutrición no se encontró evidencia significativa del tiempo por haber recibido leche materna y la presencia de la enfermedad; además se observa que no hay presencia de patologías crónicas.

Por otro lado, los hábitos comportamentales no presentaron ningún tipo de factor de protección, sin ser este resultado una conclusión válida en la investigación.

Es de resaltar que durante el análisis no se encuentran factores de riesgo con respecto al padecimiento de IRA, por el contrario se hallan factores protectores estadísticamente significativos relacionados con características sociodemográficas y nutricionales. Según lo anterior El tener un ingreso menor de un salario mínimo legal mensual (OR de 0,49 con un IC 95\% 0,24 - 0,97 p 0,039) representa un probable factor protector de los menores; lo cual no corresponde a lo descrito por la investigación tomada de AIEPI en Sao Pablo Brasil(11) el cual presenta como una asociación 
inversa donde, a menor renta familiar mayor es la prevalencia de IRA con un 51,9\% $\mathrm{y}$ una $\mathrm{p}$ de 0,008 .

También se observa que en el municipio de Suaza, el 78,9\% (OR 0,93 IC: 0,45-1,93) de la población menor de cinco años no asiste a una guardería, aunque no se presenta como un factor protector estadísticamente significativo, la presencia de las madres en el cuidado de los menores y la ocupación de ama de casa disminuye el aumento de solvencia financiera.

En un estudio realizado en México(12) la relación de las condiciones económicas de la familia ha hecho que ambos padres trabajen, aumentando la asistencia de niños a guarderías. Según la revista española de salud pública ${ }^{(13)}$ la asistencia a este tipo de establecimientos, presenta un incremento de riesgo de infección respiratoria alta $(\mathrm{RR}=$ $1,88)$, otitis media aguda $(R R=1,58)$, otitis media con derrame $(R R=2,43)$, infecciones respiratorias bajas (globalmente $R R=2,10$; neumonías $R R=1,70$; bronquiolitis $R R=1,80$; bronquitis $R R=2,10$ ).

A nivel de características nutricionales, el presentar un bajo peso al nacer (OR 0,48 IC $0,26-0,87$, p 0,01), también es considerado un probable factor protector, no existen estudios que confirmen este resultado, ya que por el contrario, según $\operatorname{Daza}^{(14)}$, el bajo peso al nacer, corresponde a un problema de salud pública, y es un indicador de salud materno-infantil, que puede contribuir a problemas de salud de corto y largo plazo.

La OPS señala como factores de riesgo para neumonía, bajo peso al nacer, desnutrición, falta de lactancia materna, polución ambiental, hacinamiento, falta de vacunación entre otras.

Aunque las variables anteriormente nombradas se tomaron en cuenta para la realización de la investigación, no se presentan datos estadísticamente significativos para la inclusión como factores de riesgo; por lo tanto, no se puede realizar un plan de acción para intervención en salud pública que plantee soluciones de intervención hacia la infección respiratoria aguda. Como consecuencia de esto, no se cumple el tercer objetivo planteado en la investigación.

\section{Conclusiones}

Se plantean las siguientes conclusiones según los hallazgos del estudio:

Los niños mayores de siete meses presentan mayor incidencia de infección respiratoria aguda que los menores de seis meses; por otro lado, el género masculino fue el sexo predominante en la investigación, donde los niños encuestados pertenecen en su mayoría al régimen subsidiado.

Más de la mitad de las madres de los menores presentan edades promedio de 21 a 30 años, sin evidencia significativa de mujeres con analfabetismo. Se evidencia que la mayoría de la población subsiste con un ingreso menor al salario mínimo legal mensual.

En el análisis se encontró que la mayor procedencia fue la zona rural, las personas de este sector residen en casas de bahareque y pisos de cemento y tierra; por otra parte dependiendo de la vereda, se observa la carencia de uno o más servicios públicos. La forma de eliminación de basuras es quemar o botar al río, lote o zanja. Es de observar que la mayoría utilizan la leña para cocinar y solo una mínima parte lo realizan con gas.

Por otro lado, la cabecera municipal y centros poblados, disfrutan de viviendas con paredes de bloque o ladrillo y pisos de cemento; por el contrario las otras partes ya descritas, gozan de todos los servicios públicos y la recolección de basuras por el carro del aseo.

La mayoría de la población reside de 1 a 8 personas por vivienda, y casi en igual proporción las viviendas constan de 1 a 3 habitaciones. En una misma vivienda residen de 1 a 3 niños, en general los niños no asisten a la guardería, pero aunque se quedan en casa pocos presentan contacto con personas fumadoras.

Las madres presentaron menos de 2 embarazos previos, y durante la etapa de gestación no se demostraron inconvenientes que pudieran afectar la salud del menor, de esta manera nacen a-término y por parto vaginal, con peso adecuado y presentando en la tabla de crecimiento y desarrollo percentiles mayores al 50. No hay ningún 
tipo de enfermedad crónica y tienen hábitos comportamentales adecuados.

Se puede decir que la incidencia es mayor en la zona rural con un 32\% de atenciones por infección respiratoria aguda en la población.

Se encuentran diferencias estadísticamente significativas de los casos y controles hacia factores protectores tales como presentar un ingreso menor al salario mínimo legal mensual y bajo peso al nacer.

No se presentan diferencias epidemiológicamente significativas para factores de riesgo de infección respiratoria aguda, por lo tanto no se cumple el tercer objetivo de la investigación.

Debido a no presentar diferencias epidemiológicamente significativas de factores de riesgo, no se puede realizar un plan de acción, que plantee soluciones para la intervención de IRA. Es necesario un nuevo estudio, donde se prolongue el tiempo de investigación aumentando el tamaño de la muestra.

\section{Agradecimientos}

Los autores desean agradecer al personal del Hospital Nuestra Señora de Fátima, al personal que colaboró con la validación y aplicación del instrumento, a las madres y acudientes de los menores y a los miembros de la Especialización en Epidemiología de la Universidad Surcolombiana.

\section{Referencias}

1. Moreno L.B, Ferrero F., Brosotti C., et al. (2010). Diseño de una regla de predicción simple para la presunción de etiología en niños con neumonía. En: Revista de la Facultad de Ciencias Médicas, Córdoba. 67(1):24.

2. Instituto Nacional de Salud. (2010). Protocolo de vigilancia de la infección respiratoria grave y enfermedad similar a la influenza (IRAG - ESI), p. 48.

3. Bermúdez L.A., Ramírez E.A., Valdés A.P. (2009). Factores de riesgo asociados a la ocurrencia de otitis media supurativa en menores de cinco años, Ese Carmen Emilia Ospina, agosto-octubre 2008. Neiva, Trabajo de grado (Epidemiología). Universidad Surcolombiana, Facultad de Salud, área de epidemiología, p. 60.

4. Jaimes M.B., Cáceres D.C., De La Hoz F., Gutiérrez C., Herrera D., et al. (2003). Factores de riesgo de infección respiratoria aguda baja grave en Bogotá, 2001. En: Rev. Biomédica, Bogotá. 23(3):287.

5. Secretaria de salud del Huila. (2008). Boletín Epidemiológico del Huila. Gobernación del Huila, p. 94.

6. Dirección Local de Salud. (2010). Perfil Epidemiológico del Municipio de Suaza. Alcaldía Municipal, p. 1-68.

7. Instituto Nacional de Salud. (2010). Protocolo de vigilancia de la infección respiratoria grave y enfermedad similar a la influenza (IRAG - ESI), p. 48.

8. Gómez P.R.M., Cruz M.J.C, Hernández R.O, Reyes M. (2003). Infecciones respiratorias agudas tratadas en la comunidad. EN: Rev Arch Méd, 7(1):6.

9. Moore H.C, De Klerk N., Richmond P., et al. (2010). A retrospective population-based cohort study identifying target areas for prevention of acute lower respiratory infections in children. En: Revista BMC Public Health, 10:757.

10. Dane. Proyecciones municipales 2005-2011. En: Dane. Información estadística [en línea]. (2005). [consultado 11 jun. 2010]. Disponible en http://www.dane.gov.co/index.php? Itemid $=995 \& \mathrm{id}=497 \&$ option=com_content \&sectionid $=16 \&$ task $=$ category

11. Freitas A.J., Baptista M.A., Halpern R., et al. (1998). Prevalencia y factores de riesgo de infección respiratoria aguda en niños de seis meses de edad en Pelotas, Río Grande do Sul. En: Organización Panamericana de Salud (PAHO). p. 236. ISBN 927532212.

12. Nandi-Lozano E., et al. (2002). Infección respiratoria aguda en niños que acuden a un Centro de Desarrollo Infantil. En: Salud Pública Méx, Cuernavaca, 44(3):

13. Ochoa C.; Barajas M.V; Muñoz B. (2007). Relación entre la asistencia a guarderías y enfermedad infecciosa aguda en la infancia: una revisión sistemática. En: Rev. Esp. Salud Pública, Madrid, 81(2):121.

14. Daza V., Jurado W., Duarte D., et al. (2009). Bajo peso al nacer: exploración de algunos factores de riesgo en el Hospital Universitario San José en Popayán (Colombia). En: Rev. colombiana. obstet. Ginecol, Popayán, 60(2): 124-134. 